\title{
Universal Phenomena in Solution Bifurcations of Some Boundary Value Problems ${ }^{\dagger}$
}

\author{
Alexander SHARKOVSKY and Andrij SIVAK \\ Institute of Mathematics of the National Ukrainian Academy of Sciences, \\ Tereshchenkivska Street 3, 252004 Kiev, Ukraina
}

Received April 10, 1994

\begin{abstract}
We show that for a class of boundary value problems, the space of initial functions can be stratified dependently on the limit behavior (as the time variable tends to infinity) of solutions. Using known results on universal phenomena appearing in bifurcations of one parameter families of one-dimensional maps, we establish that, for certain types of boundary value dependence, a similar quantitative and qualitative universality is also observed in the stratification and bifurcations of solutions.
\end{abstract}

\section{Introduction}

In this paper we describe universal phenomena (including quantitative ones) in bifurcations of solutions of boundary value problems, which are generated by the universality in one parameter families of one-dimensional maps. This universality is based on the following properties of one-dimensional maps. It is known since sixtieth years that for the continuous maps of the real line into itself, the coexistence of periods of cycles of such maps obeys the following ordering of the natural numbers [10]:

$$
\begin{aligned}
1 \prec & 2 \prec 2^{2} \prec 2^{3} \prec \ldots \\
& \prec 7 \cdot 2^{2} \prec 5 \cdot 2^{2} \prec 3 \cdot 2^{2} \prec \ldots \prec 7 \cdot 2 \prec 5 \cdot 2 \prec 3 \cdot 2 \prec \ldots \prec 7 \prec 5 \prec 3
\end{aligned}
$$

namely, if for some $n \geq 1$, a continuous map $f$ of the real line has a cycle of period $n$ and $m \prec n$, then $f$ has also a cycle of period $m$. Since there are different types of cycles with the same period, it has been understood later that the ordering (1) has a certain analogue for the set of types of cycles [12]. More detailed, for any $n \geq 1$, each cycle of period $n$

Copyright (c) 1994 by Mathematical Ukraina Publisher. All rights of reproduction in any form reserved.

\footnotetext{
${ }^{\dagger}$ The research was made possible in part by Grant N V6G000 from the International Science Foundation.
} 
define a map on the $n$-point set considered according to the linear order on the real line or, in other words, a cyclic permutation of integers $1,2, \ldots n$ that provides an equivalence relation between cycles of the same period (this idea was treated in various contexts by many workers). Since the number of different cyclic permutations of $n$ points on the real line is finite for each $n$, we have a finite number of different types for cycles of the same period; these types will be denoted by $(n, i)$ where $n$ is the period of the cycle and $i \in\left\{1, \ldots, k_{n}\right\} ; k_{n}$ depends on the class of maps under consideration. If we consider the class of unimodal maps only, then the analogue of the ordering (1) for types of cycles still remains to be linear. (Recall that a continuous map is called unimodal if it has just one turning point, i.e., the point where the map has a local extremum and hence it consists of two monotone branches; for the sake of definiteness, this unique extremal point will be supposed to be a maximum and denoted by $c$.) For the class of unimodal maps, we have either $(n, i) \prec(m, j)$ or $(m, j) \prec(n, i)$ for any $n, m \geq 1$ and $i \in\left\{1, \ldots k_{n}\right\}, j \in\left\{1, \ldots k_{m}\right\}$ : the set of all types of cycles of unimodal maps obeys the ordering

$$
\begin{aligned}
(1,1) \prec(2,1) \prec\left(2^{2}, 1\right) & \prec\left(2^{3}, 1\right) \prec \ldots \\
\prec(5 \cdot 2,1) \prec \ldots \prec & (3 \cdot 2,1) \prec(12,2) \prec \ldots \prec(5,1) \prec \ldots \prec(3,1) \prec(6,2) \prec \ldots \\
& \prec\left(4, k_{4}\right) \prec \ldots \prec\left(5, k_{5}\right) \prec \ldots \prec\left(6, k_{6}\right) \prec \ldots \prec\left(7, k_{7}\right) \prec \ldots
\end{aligned}
$$

where " $\prec$ " has the same sense as in (1).

Remarks 1. Any $\left(n, i^{\prime}\right)$ in $(2)$ is followed immediately by $\left(2 n, i^{\prime \prime}\right)$ but there are types in (2) having no immediate predecessor.

2. The ordering for the subset $\{(n, 1)\}_{n=1}^{\infty}$ of types repeats the ordering (1).

3 . The ordering (2) remains true in the space of all continuous maps of the real line if we consider no other types of cycles additionally.

4. The ordering (1) provides a simple criterion for the complexity of one-dimensional maps: it is natural to call a map "the simplest" if it has only fixed points and "the most complicated" if it has a cycle of period 3. A similar criterion of complexity can be based on the ordering (2).

If we consider a family of unimodal maps continuously depending on a parameter $p$, then we can observe the appearance of cycles of various periods in accordance with (1) and, if we use information about types of cycles, the appearance of various types of cycles in accordance with (2) as the parameter varies. There are a lot of families of unimodal maps, for which the appearance of cycles of all types can be observed for a finite interval of the parameter. The simplest and most remarkable of them are quadratic families a representative of which may be $F_{p}(x)=-x^{2}+p$. For the quadratic families, the simplest decomposition of the parameter space with respect to various types of dynamical behavior takes place and we use this family for our explanations in what follows in order to avoid unimportant details.

Let $p(n, i)$ denote the greatest lower bound for the set of the parameters $p$ when the map $F_{p}: x \rightarrow-x^{2}+p$ has a cycle of type $(n, i)$ where $(n, i)$ is from $(2)$. Then by $(2)$ we must have

$$
\begin{aligned}
p(1,1)<p(2,1)<p\left(2^{2}, 1\right) & <p\left(2^{3}, 1\right)<\ldots \\
<p(5 \cdot 2,1) & <\ldots<p(3 \cdot 2,1)<\ldots<p(5,1)<\ldots<p(3,1)<p(6,2)<\ldots \\
& <p\left(4, k_{4}\right)<\ldots<p\left(5, k_{5}\right)<\ldots<p\left(6, k_{6}\right)<\ldots<p\left(7, k_{7}\right)<\ldots
\end{aligned}
$$


(Note that (3) holds for a much larger class of families of unimodal maps; it is necessary to require the smoothness of the maps and the parameter dependence.)

For any type $(n, i)$ there is an (open or semi-open) interval $A(n, i)$ of the parameter values such that the map $F_{p}: x \rightarrow-x^{2}+p$ has an attracting cycle of the type $(n, i)$. For a large class of families including the quadratic family, these intervals are mutually disjoint and the points $p(n, i)$ are the left ends of these interval. All intervals $A(n, i)$ belong to the interval $P=\left[p_{\min }, p_{\max }\right]$ in the parameter space where $p_{\min }=p(1,1)$ and $p_{\max }=$ $\lim _{n \rightarrow \infty} p\left(n, k_{n}\right)$; in the particular case of the quadratic family $F_{p}(x)=-x^{2}+p$ we have $P=$ $\left[-\frac{1}{4}, 2\right]$. The interval $P$ in the parameter space is characterized by the property that, for any parameter value $p \in P$, the map $F_{p}$ has a finite invariant interval. It has been recently proved [16] that the set of parameters, for which corresponding maps in the quadratic family have an attracting cycle, being contained in the interval $P$, contains an open dense set in $P$. On the other hand, by the earlier result [6] the set of parameters corresponding to maps with absolutely continuous invariant measure has a positive Lebesgue measure (although, according to the above mentioned result from [16], this set is nowhere dense in $P$ because it belongs to the complement of the set of parameters when attracting cycles exist). This shows that from the metric viewpoint, the complex dynamical behavior is not exceptional in the quadratic family.

Since the interval $P$ is bounded, we can define $p\left(2^{\infty}, 1\right)=\lim _{n \rightarrow \infty} p\left(2^{n}, 1\right)$. By a straightforward calculations we can see also that values $p\left(2^{n}, 1\right), n=0,1, \ldots$, converge geometrically:

$$
\lim _{n \rightarrow \infty} \frac{p\left(2^{n}, 1\right)-p\left(2^{n-1}, 1\right)}{p\left(2^{n+1}, 1\right)-p\left(2^{n}, 1\right)}=\delta
$$

where $\delta$ is the constant equal to $4.669 \ldots$ (Note that the interval $A\left(2^{n}, 1\right)$ corresponding to the existence of the attractive cycle of type $\left(2^{n}, 1\right)$ for $F_{p}$, has its ends at the points $p\left(2^{n}, 1\right)$ and $p\left(2^{n+1}, 1\right)$. Hence the constant $\delta$ characterizes also the ratio $\operatorname{mes}\left(A\left(2^{n}, 1\right)\right) / \operatorname{mes}\left(A\left(2^{n+1}, 1\right)\right)$ as $n \rightarrow \infty$ where mes(.) denotes the Lebesgue measure of a set.) For the limit parameter value $p=p\left(2^{\infty}, 1\right)$, the map $F_{p}$ has a minimal Cantorlike set, which is the closure of the trajectory of the critical point $c=0$ and which attracts trajectories of almost all (with respect to the Lebesgue measure) points in $I_{0}(p)$. This Cantor-like set is self-similar and a quantitative characterization of this self-similarity is reflected by the relation

$$
\lim _{n \rightarrow \infty} \frac{F_{p}^{2^{n}}(c)-c}{c-F_{p}^{2^{n+1}}}=\alpha
$$

for $p=p\left(2^{\infty}, 1\right)$, where $\alpha$ is the constant equal to $2.502 \ldots$

Feigenbaum [4] and Collet and Tresser [1] were the first who numerically observed that the constants $\delta$ and $\alpha$ are universal: they discovered independently that these constants are the same for many different one parameter families of unimodal maps. Later similar quantitative universalities were established for other sequences of parameter values in (3), for example, it was observed that

$$
\lim _{n \rightarrow \infty} \lim _{k \rightarrow \infty} \frac{\operatorname{mes}\left(A\left((2 k+1) 2^{n}, 1\right)\right)}{\operatorname{mes}\left(A\left((2 k+3) 2^{n}, 1\right)\right)}=\gamma
$$

where $\gamma=2.905 \ldots$ is one more universal constant (see [7]). The first rigorous proof of 
the universal phenomena (4) and (5) was obtained by Lanford [8]. For further references see [3] and [2].

At last, each value $p(n, i)$ in (3) corresponds to the interval $P(n, i)$, which is characterized by the property that, for any $p \in P(n, i)$, besides a cycle of type $(n, i)$, the map $F_{p}$ has an interval cycle of type $(n, i)$. (Similarly to the definition of a cycle, an interval cycle is defined to be a finite collection of mutually disjoint intervals cyclically mapped by the mapping each into another; also similarly to the case of cycles, the type of the interval cycle can be defined.) In particular, we have $P(1,1)=P, P(2,1)=(p(2,1), \bar{p}(2,1)) \subset P(1,1)$ where $\bar{p}(2,1)=\lim _{k \rightarrow \infty} p(2 k+1,1), \ldots, P\left(2^{n}, 1\right)=\left(p\left(2^{n}, 1\right), \bar{p}\left(2^{n}, 1\right)\right) \subset P\left(2^{n-1}, 1\right)$ where $\bar{p}\left(2^{n}, 1\right)=\lim _{k \rightarrow \infty} p\left((2 k+1) 2^{n-1}, 1\right)$, and so on. Note that the point $p\left(2^{\infty}, 1\right)$ is the intersection of all $P\left(2^{n}, 1\right)$, and the length of $P\left(2^{n+1}, 1\right)$ is reduced by the factor $\delta$ with respect to the length of $P\left(2^{n}, 1\right)$ as $n \rightarrow \infty$.

In the present paper we develop the results of [15] and show that similar quantitative and universal phenomena can be observed in solution bifurcations of some boundary value problems when initial or boundary conditions vary.

\section{Reducible boundary value problems}

There is a class of boundary value problems, which can be reduced to difference equations. We consider the following example of coupled partial differential equations with boundary and initial conditions:

$$
\begin{aligned}
& \frac{\partial u}{\partial t}-a \frac{\partial u}{\partial x}=0, \\
& \frac{\partial v}{\partial t}+b \frac{\partial v}{\partial x}=0, \quad x \in[0,1], t \geq 0
\end{aligned}
$$

where $a, b>0$;

$$
\begin{aligned}
& u=v \quad \text { at } x=0, \\
& \frac{\partial u}{\partial t}=f(v) \frac{\partial v}{\partial t} \quad \text { at } x=1, \\
& u(x, 0)=s_{1}(x) \quad \text { and } \quad v(x, 0)=s_{2}(x) .
\end{aligned}
$$

Solutions of the boundary value problem (7)-(9) are the functions $u(x, t)=y_{1}\left(t+\frac{x}{a}\right)$, $v(x, t)=y_{2}\left(t-\frac{x}{b}\right)$, which satisfy both boundary and initial conditions. Due to (8) the problem of determination of such functions can be reduced to the difference-differential equation

$$
y^{\prime}(t)=f(y(t-1)) y^{\prime}(t-1)
$$

under the initial conditions

$$
y(t)=s(t), \quad t \in[0,1]
$$

where $s(t)$ is defined by the initial conditions (9):

$$
s(t)=\left\{\begin{array}{ll}
s_{2}\left(1-\frac{a+b}{a} t\right) & \text { if } t \in[0, a /(a+b)] \\
s_{1}\left(\frac{a+b}{b} t-\frac{a}{b}\right) & \text { if } t \in[a /(a+b), 1]
\end{array} .\right.
$$


It should be noted that equations of the type (10) were studied in [9], [11].

The problem (10)-(11) is reduced to the following difference equation with continuous argument

$$
y(t)=F(y(t-1))+p, \quad p=s(1)-F(s(0))
$$

under the initial conditions

$$
y(t)=s(t), \quad t \in[0,1),
$$

where $F$ is a primitive function for $f$; we will assume that $F(0)=0$.

We see that equation (13) depends on the parameter $p=p(s)=s_{1}(1)-F\left(s_{2}(1)\right)$ which is determined by the initial conditions (9). The limit behavior of solutions of the difference equation (13) with continuous argument depends essentially on the dynamics of the corresponding map from the one parameter family

$$
y \rightarrow F(y)+p
$$

of the real line into itself, and since the dynamics of the map varies as the parameter $p$ varies, the asymptotic behavior of solutions of the boundary value problem (7)-(9) is also depending on the initial conditions (9) according to the dependence of the dynamics on the parameter in the family (14).

Note that if the second boundary condition in (8) is replaced by the condition

$$
u=F(v)+p,
$$

where $p$ is a parameter, then evidently the boundary value problem can be also reduced to the difference equation (13) but in this case the new parameter $p$ is independent from the initial data. Note also that such method of reduction of boundary value problems to difference, difference-differential or difference-integral equations has been studied in [13].

\section{Stratification of the space of initial functions}

Let us consider equation (10) which the problem (7) - (9) is reduced to. It is clear that properties of any solution of the problem $(7)-(9)$, including boundedness, periodicity in $t$ and other ones, are determined by properties of the corresponding solution of the problem (10)-(11). Every initial function $s(t)$ of the form (12), $t \in[0,1]$, defines a unique solution $y_{s}(t), t \geq 0$, of the equation (10). As we have seen above, the behavior of this solution depends essentially on the value $p(s)=s(1)-F(s(0))=s_{1}(1)-F\left(s_{2}(1)\right)$.

Let us suppose that the function $f$ in the boundary conditions (8) of the boundary value problem (7)-(9) belongs to the class $C^{2}\left(R^{1}, R^{1}\right)$ of twice continuously differentiable functions of the real line $R^{1}$ and satisfies the following two conditions:

(A) $f(0)=0$ and $f^{\prime}(y)<-\varepsilon$ for some fixed $\varepsilon>0$ and for all $y$;

(B) $y f^{\prime \prime}(y) \geq 0$ for all $y$.

An example of the function satisfying these conditions may be any linear function of the form $f(y)=-k y$ where $k>0$.

Under these conditions on the function $f$, any map from the one parameter family $F_{p}(y)=F(y)+p(s)$ (see (14)) belongs to the class $C^{3}\left(R^{1}, R^{1}\right)$ and satisfy the following conditions: 
(i) $F_{p}$ is unimodal, i.e., it has just one point $c$ at which the derivative of $F_{p}$ vanishes and alternates its sign (by the condition (A) above we have $c=0$ in this case);

(ii) $F_{p}^{\prime \prime}(y)<-\varepsilon$, i.e., the map $F_{p}$ is convex;

(iii) $F_{p}$ has negative Schwarzian derivative, i.e.,

$$
\frac{F_{p}^{\prime \prime \prime}(y)}{F_{p}^{\prime}(y)}-\frac{3}{2}\left(\frac{F_{p}^{\prime \prime}(y)}{F_{p}^{\prime}(y)}\right)^{2}<0 \text { for } y \neq c .
$$

The condition (iii) guarantees that the unimodal map $F_{p}$ has at most one attractor, and the condition (ii) together with (iii) imply that the dynamics of $F_{p}$ becomes more complicated as the parameter $p$ increases.

For the family $F_{p}$ satisfying the conditions (i)-(iii), the following conclusions can be derived. Since $F_{p}(y)=F(y)+p$ is a family of unimodal maps and the parameter $p$ is included additively into the family, there exist finite $p_{\min }=p(1,1)$ and $p_{\max }=\lim _{n \rightarrow \infty} p\left(n, k_{n}\right)$; $p_{\min }$ is defined by the system of equations $y=F(y)+p$ and $F^{\prime}(y)=1$ and corresponds to the appearance of the fixed point; $p_{\max }$ is defined by the equation $F(p)=F(p+F(p))$ (but $F(p) \neq 0$ ) and corresponds to the case when the point $y=0$ hits the left fixed point in two steps $\left(0 \rightarrow p \rightarrow p+F(p) \rightarrow p+F(p+F(p))=p+F(p) \rightarrow \ldots\right.$, i.e., $\left.F^{2}(0)=F^{3}(0)\right)$.

As has been mentioned above, each $p \in P=\left[p_{\min }, p_{\max }\right]$ corresponds to a bounded invariant interval $I(p)=\left[a_{p}, a_{p}^{\prime}\right]$ where $a_{p}$ is the left one of two fixed points (i.e., the least of two roots of the equation $y=F(y)+p$ ), and $a_{p}^{\prime}$ is its preimage (i.e., a root of the equation $F\left(a_{p}\right)+p=a_{p}^{\prime}$ ). If $p<p_{\min }$, then $F_{p}^{n}\left(y_{0}\right) \rightarrow-\infty$ for any $y_{0}$ as $n \rightarrow \infty$; if $p>p_{\max }$, then $F_{p}^{n}\left(y_{0}\right) \rightarrow-\infty$ for any $y_{0}$ except for points from a Cantor set of zero Lebesgue measure; if $p \in P$ but $y_{0} \notin I(p)$, then in this case we have $F_{p}^{n}\left(y_{0}\right) \rightarrow-\infty$ as well. Therefore a solution of the difference equation $(13),\left(11^{\prime}\right)$ is bounded only if the initial function $s(t)$ satisfies the conditions $p(s)=s(1)-F(s(0)) \in P$ and $I_{s(t)} \subset I(p(s))$, where $I_{s(t)}=\{y \mid y=s(t), \quad t \in[0,1]\}$. (Note that for any $p>p_{\max }$, there still exist two bounded solutions $y(t) \equiv a$ where $a$ is one of two fixed points of $F_{p}(y)$, i.e., of the roots of the equation $a=F(a)+p$.)

In the space of initial functions (12) we consider the subset

$$
\mathcal{P}=\left\{s \in C^{1}([0,1]): p(s) \in P \quad \text { and } \quad s(t) \in I(p(s)) \quad \text { for } \quad t \in[0,1]\right\}
$$

where $I(p(s))$ denotes the maximal bounded invariant interval of $F_{p(s)}$. Then the set $\mathcal{P}$ consists of the initial functions generating bounded solutions of the problem (7)-(9). Note that the set $\mathcal{P}$ is "massive" because it contains an open set (with respect to the uniform metrics in $C([0,1]))$.

Considering the continuous map $p: C([0,1]) \rightarrow R^{1}$, which is defined by the equality $p=s_{1}(1)-F\left(s_{2}(1)\right)$, we obtain a stratification of the set of initial functions $\mathcal{P}$ of the boundary value problem (7)-(9) induced by the stratification of the space of parameter values of the family $F_{p}$ described in the introductory section. Moreover, the map $p$ : $C([0,1]) \rightarrow R^{1}$ generate a mesure $\mu$ on the set of initial functions defined for preimages of Lebesgue measurable sets $L \in R^{1}$ by the equality $\mu\left(p^{-1}(L)\right)=\operatorname{mes}(L)$ where $\operatorname{mes}(L)$ denote the Lebesgue measure of $L$. Using this measure $\mu$ and the universality for onedimensional maps, we can estimate "the thickness" of the strata in the space of initial functions. 
The following theorem is an example of a rigorous result in this direction. Using the notation from the introductory section, we define

$$
\mathcal{P}\left(2^{n}, 1\right)=\left\{s \in \mathcal{P}: p(s) \in P\left(2^{n}, 1\right)\right\}
$$

and

$$
\mathcal{A}\left((2 m+1) 2^{n}, 1\right)=\left\{s \in \mathcal{P}: p(s) \in A\left((2 m+1) 2^{n}, 1\right)\right\}
$$

$n=0,1,2 \ldots$ and $m=0,1,2, \ldots$ We have that $\mathcal{P}=\mathcal{P}(1,1) \supset \mathcal{P}(2,1) \supset \mathcal{P}\left(2^{2}, 1\right) \supset \ldots$ and $\mathcal{A}(i, 1), i=1,2, \ldots$, are mutually disjoint subsets of $\mathcal{P}$. (Note that each of these sets has a nonempty interior.)

Theorem 1 With the universal constants $\delta=4.669201 \ldots$ and $\gamma=2.94805 \ldots$, the following asymptotic formulas are true:

$$
\lim _{n \rightarrow \infty} \frac{\mu\left(\mathcal{P}\left(2^{n}, 1\right)\right)}{\mu\left(\mathcal{P}\left(2^{n+1}, 1\right)\right.}=\lim _{n \rightarrow \infty} \frac{\mu\left(\mathcal{A}\left((2 m+1) 2^{n}, 1\right)\right)}{\mu\left(\mathcal{A}\left((2 m+1) 2^{n+1}, 1\right)\right)}=\delta
$$

(where $m \geq 1$ is fixed) and

$$
\lim _{n \rightarrow \infty} \lim _{m \rightarrow \infty} \frac{\mu\left(\mathcal{A}\left((2 m+1) 2^{n}, 1\right)\right)}{\mu\left(\mathcal{A}\left((2 m+3) 2^{n}, 1\right)\right)}=\gamma
$$

Similarly to the sets $\mathcal{A}\left((2 m+1) 2^{n}, 1\right)$ we can also define the sets

$$
\mathcal{A}(l, i)=\{s \in \mathcal{P}: p(s) \in A(l, i)\}
$$

and the sets

$$
\mathcal{P}(l, i)=\{s \in \mathcal{P}: p(s) \in P(l, i)\}
$$

for all $l \geq 1$ and $i=1, \ldots k_{l}$, and formulate corresponding statements estimating measures of these sets.

Using theorem 1 and the results represented in the next section, we can see that theorem 1 provides an estimate for sets of initial functions generating solutions of the boundary value problem (7)-(9) in dependence on various types of limit behavior.

\section{Asymptotic properties of solutions}

Solutions of the boundary value problem (7)-(9) are single-valued smooth functions defined in the semi-strip $D=\{0 \leq x \leq 1\} \times\{t \geq 0\}$. Nevertheless the function which describes the limit behavior of a solution may be nonsmooth and even may not be single-valued (see [13] for more details). Therefore we complete the space $C^{1}(D)$ of continuously differentiable functions on $D$ containing the solutions of the problem (7)-(9) by many-valued functions on $D$, values of which at points of the semi-strip $D$ are either a point or a closed subset of the real line $R^{1}$ (it is sufficient to consider only the functions, the values of which are either a point or a closed interval). This extended space is denoted by $C^{+}(D)$.

In order that the distance between points of the space $C^{+}(D)$ can be measured, we use the Hausdorff distance between graphs of the functions representing these points: if $\Gamma_{1}$ 
and $\Gamma_{2}$ denote graphs of two functions from $C^{+}(D)$, then the Hausdorff distance between these graphs is defined to be

$$
\Delta\left(\Gamma_{1}, \Gamma_{2}\right)=\max \left\{\sup _{z \in \Gamma_{1}} \rho\left(z, \Gamma_{2}\right), \sup _{z \in \Gamma_{2}} \rho\left(z, \Gamma_{1}\right)\right\}
$$

where, as is customary, $\rho(z, B)$ denotes the distance from the point $z$ to the set $B$.

In order to describe the limit behavior of a solution of the problem (7)-(9), we use the following definition.

Definition Let $w_{1} \in C(D)$ and $w_{2} \in C^{+}(D)$. We say that $w_{2}$ is a limit function for $w_{1}$ as $t \rightarrow \infty$ if

$$
\Delta\left(\Gamma^{T}\left(w_{1}(x, t)\right), \Gamma^{T}\left(w_{2}(x, t)\right)\right) \rightarrow 0, \quad \text { as } T \rightarrow \infty,
$$

where $\Gamma^{T}(\cdot)$ denotes the graph of a function for $t \geq T$. Functions from $C^{+}(D)$ which are limit functions for solutions of the boundary value problem (7)-(9) are called generalized solutions of the problem (7)-(9).

As is customary, we say that a function $w(x, t) \in C^{+}(D)$ is periodic with period $\nu$ in $t$ if $w(x, t+\nu)=w(x, t)$ for all $t \geq 0, x \in[0,1]$.

Since any solution of the boundary value problem (7)-(9) has the specific form $u(x, t)=$ $y\left(t+\frac{x}{a}\right), v(x, t)=y\left(t-\frac{x}{b}\right)$ where $y(t)$ is a solution of the difference-differential problem (10)-(11), the limit behavior of solutions of the problem (7)-(9) is described by the limit behavior of corresponding solutions of the problem (10)-(11). Therefore we consider the family (14) and use the idea of prolongations of trajectories (see, for example, [11]) in order to understand the limit behavior of solutions: for any point $y$ in the maximal invariant interval $I(p)$ of the map $F_{p}$, we set

$$
F_{p}^{*}(y)=\bigcap_{\varepsilon>0} \bigcap_{j \geq 0} \overline{\bigcup_{i \geq j} F_{p}^{i !}\left(U_{\varepsilon}(y)\right)}
$$

where, as is customary, $U_{\varepsilon}(y)$ denotes the $\varepsilon$-neighborhood of the point $y$ in the interval $I_{p}$, the overlined set denotes the closure of this set, $i !=1 \cdot 2 \cdot \ldots \cdot i$ and $0 !=1$.

The equality (17) defines a map $F_{p}^{*}: I(p) \rightarrow 2^{I(p)}$ and the asymptotic behavior of a solution $y_{s}(t)$ of the difference-differential equation (10) corresponding to an initial function $s(t)$ that can be characterized by the function $y_{s}^{*}(t)$, which is defined by the equality

$$
y_{s}^{*}(t)=F_{p}^{i}\left(F_{p}^{*}(s(t-i))\right) \text { for } t \in[i, i+1),
$$

$i=0,1,2, \ldots$. More exactly, by using the Hausdorff distance for graphs of functions, we have

$$
\Delta\left(\Gamma^{T}\left(y_{s}(t)\right), \Gamma^{T}\left(y_{s}^{*}(t)\right)\right) \rightarrow 0, \quad \text { as } T \rightarrow \infty,
$$

where $\Gamma^{T}(y(t))$ denotes the graph of a function $y(t)$ for $t \geq T$.

The following two propositions consider sets of initial functions which generate solutions characterized respectively by limit generalized solutions of quite different types (see, for example, [13]).

Proposition 1 In the set $\mathcal{P}$ of initial functions generating bounded solutions of the boundary value problem (7)-(9), there is an open dense subset $\mathcal{P}_{+}$such that for any $s(t) \in \mathcal{P}_{+}$the corresponding solution $u(x, t), v(x, t)$ of the problem $(7)-(9)$ tends (in the 
Hausdorff metrics for graphs) as $t \rightarrow \infty$ to a generalized periodic solution $u^{*}(x, t), v^{*}(x, t)$ (with a period of the form $m \cdot \omega$ where $m$ is a positive integer and $\omega=\frac{1}{a}+\frac{1}{b}$ ). This limit solution, in turn, is single-valued at almost all points of the semi-strip $D$ (from both an category and the Lebesgue measure points of view).

On the other hand, due to the above mentioned result of [6], for the induced measure $\mu$ on the set of initial functions, which has been defined above by the relation $p(s)=$ $s(1)-F(s(0))$, we have $\mu\left(\mathcal{P} \backslash \mathcal{P}_{+}\right)>0$. Hence the set of initial functions, which generate solutions of the boundary value problem (7)-(9) characterized by "random" asymptotic behavior, is not exceptional from the metric viewpoint. Namely, the following statement takes place.

Proposition 2 In the set $\mathcal{P}$ of initial functions generating bounded solutions of the boundary value problem (7)-(9), there is a subset $\mathcal{P}_{\sigma}$ of positive measure $\mu$ such that for any $s(t) \in \mathcal{P}_{\sigma}$ the corresponding solution $u(x, t), v(x, t)$ of the boundary value problem (7)-(9), tends as $t \rightarrow \infty$ (in the Hausdorff metrics for graphs), to a generalized periodic solution $u^{*}(x, t), v^{*}(x, t)$, which is interval-valued at any point of the semi-strip $D$.

Of course, it is possible to use more sophisticated metrics in order to describe asymptotic behavior of solutions more exactly. For example, if we use the special metric from [14] involving all finite-dimensional joint distributions combined with averaging operation, then we can consider generalized periodic solutions in Proposition 2 as random functions in a certain sense.

Let us consider the sequence

$$
\mathcal{P}=\mathcal{P}(1,1) \supset \mathcal{P}(2,1) \supset \mathcal{P}\left(2^{2}, 1\right) \supset \mathcal{P}\left(2^{3}, 1\right) \supset \ldots
$$

of nested sets of initial functions, which has been defined above. Recall that for any initial function $s \in \mathcal{P}\left(2^{n}, 1\right)$, the corresponding map $F_{p}$ has an interval cycle of type $\left(2^{n}, 1\right)$. The map $F_{p\left(2^{\infty}, 1\right)}$, which corresponds to initial functions from the set $\mathcal{P}\left(2^{\infty}, 1\right)=\bigcap_{n \geq 0} \mathcal{P}\left(2^{n}, 1\right)$, has an infinite sequence of nested interval cycles of types $\left(2^{n}, 1\right), n=0,1, \ldots$, which approximate its Cantor-like attractor. Due to the above mentioned quantitative universality of the metric structure of this attractor, we can formulate the following statement, which establishes a possibility for the approximation of solutions of the boundary value problem (7)-(9) by periodic solutions.

Theorem 2 For any initial function $s(t) \in \mathcal{P}\left(2^{n}, 1\right), n \geq 0$, the corresponding solution $u(x, t), v(x, t)$ of the boundary value problem (7)-(9) can be approximated by the generalized periodic solution $u^{* *}(x, t), v^{* *}(x, t)$ of period $2^{n} \cdot \omega$ in $t$ (where $\omega=\frac{1}{a}+\frac{1}{b}$ ) such that

$$
\begin{aligned}
& \limsup _{T \rightarrow \infty} \Delta\left(\Gamma^{T}(u(x, t)), \Gamma^{T}\left(u^{* *}(x, t)\right)\right) \sim \alpha^{-n}, \\
& \limsup _{T \rightarrow \infty} \Delta\left(\Gamma^{T}(v(x, t)), \Gamma^{T}\left(v^{* *}(x, t)\right)\right) \sim \alpha^{-n},
\end{aligned}
$$

where $\alpha=2.502 \ldots$ is a universal constant (see (5)). This generalized periodic solution can be chosen such that it is single-valued at almost all points (from both the category and Lebesgue measure viewpoints) in the semi-strip D.

Due to Theorems 1 and 2 we can see that having defined initial functions approximately to within some $\varepsilon>0$, it is sufficient to determine $F_{p}^{* *}(y)$ for some $n$ such that $\alpha^{-n} \approx \varepsilon$. We 
can see also that the set of initial functions (and hence solutions as well), for which such approximation holds, is diminishing much faster than $\varepsilon$ decreases because the constant $\delta$ is greater than $\alpha$.

Note that besides the universality argument, the proof of Theorem 2 is based also on the result of [5] where it has been proved that the attractor of any unimodal map with negative Schwarzian attracts almost all points in the invariant interval of the map. Note also that analogous statement may be formulated for other infinite sequences of above defined sets $\mathcal{P}(n, i)$ of initial functions of the boundary value problem (7)-(9).

\section{An example}

Let us consider a simple example when the function $f(y)$ in the boundary conditions (8) is linear: $f(y)=-k y$ where $k>0$. In this case the boundary value problem (7)-(9) is reduced to the following difference equation with continuous argument

$$
\begin{aligned}
y(t) & =-\frac{k}{2} y^{2}(t-1)+p, \\
p & =p(s(t))=s_{1}(1)+\left(s_{2}(1)\right)^{2},
\end{aligned}
$$

which corresponds to the family of maps $F_{p}: y \rightarrow-\frac{k}{2} y^{2}+p$ and where the initial function $s(t)$ is defined by (12). For the sake of definiteness we set $k=2$.

For $p \in[-1 / 4,2]$, the interval $I(p)=[-q(p), q(p)]$, where $q(p)=1 / 2+\sqrt{1 / 4+p}$, is an invariant interval of the map $F_{p}$, i.e. $F_{p}(I(p)) \subset I(p)$. For any $y \notin I(p)$, points $F_{p}^{i}(y)$ tend to $-\infty$ as $i \rightarrow \infty$. A solution $u(x, t), v(x, t)$ of the boundary value problem (7)-(9) is bounded only if

$$
\begin{array}{r}
s(t) \in \mathcal{P}=\left\{s \in C^{1}([0,1]): s_{1}(1)+\left(s_{2}(1)\right)^{2} \in[-1 / 4,2]\right. \text { and } \\
s(t) \in[-q, q] \text { for all } t \in[0,1]\},
\end{array}
$$

where $q=1 / 2+\sqrt{1 / 4+s_{1}(1)+\left(s_{2}(1)\right)^{2}}$.

Other sets $\mathcal{P}\left(2^{n}, 1\right), n \geq 1$, and $\mathcal{A}(m, i), m, i \geq 1$, are defined similarly:

$$
\begin{array}{ll}
\mathcal{P}(1,1)=\mathcal{P}, & \mathcal{A}(1,1)=\{s \in \mathcal{P}: p(s) \in[-1 / 4,3 / 4]\}, \\
\mathcal{P}(2,1)=\{s \in \mathcal{P}: p(s) \in[3 / 4,1.543 \ldots]\}, & \mathcal{A}(2,1)=\{s \in \mathcal{P}: p(s) \in[3 / 4,5 / 4]\}, \\
\mathcal{P}(4,1)=\{s \in \mathcal{P}: p(s) \in[5 / 4,1.43 \ldots]\}, & \mathcal{A}(4,1)=\{s \in \mathcal{P}: p(s) \in[5 / 4,1.368 \ldots]\}
\end{array}
$$

and so on. Note that having defined $\mathcal{P}(1,1), \mathcal{P}(2,1)$ and several more first sets, we can determine other sets $\mathcal{P}\left(2^{n}, 1\right)$ approximately by using estimates established by theorem 1 . An analogous observation is true for the sequence of sets $\mathcal{A}\left(2^{n}, 1\right), n=0,1, \ldots$ Note also that for this example the boundary conditions (8) we have

$$
\bigcap_{i \geq 0} \mathcal{P}\left(2^{i}, 1\right)=\left\{s \in \mathcal{P}: s_{1}(1)+\left(s_{2}(1)\right)^{2}=p\left(2^{\infty}, 1\right)\right\},
$$

where $p\left(2^{\infty}, 1\right)=1.4011 \ldots$ 


\section{References}

[1] Collet P. and Tresser C., Itération d'endomorphismes et groupe de renormalisation, J. de Physique, 1978, C5, 25.

[2] Eckmann J.-P. and Wittwer P., A complete proof of Feigenbaum conjectures, J. Stat. Physics, 1987, V.46, 455-475.

[3] Epstein H., New proofs of the existence of the Feigenbaum functions, Commun. Math. Physics, 1986, V.106, 395-426.

[4] Feigenbaum M.J., Quantitative universality for a class of nonlinear transformations, J. Stat. Physics, 1978, V.19, N 1, 25-52.

[5] Guckenheimer J., Sensitive dependence to initial conditions for one-dimensional maps, Commun. Math. Physics, 1979, V.70, 133-160.

[6] Jakobson M.V., Absolutely continuous invariant measures for one-parameter families of onedimensional maps, Commun. Math. Phys., 1981, V.81, 39-88.

[7] Kolyada S.F. and Sivak A.G., Universal constants for one parameter families of maps, in: Oscillation and Stability of Solutions of Functional-Differential Equations, Kiev, Inst. Math. Ukrain. Acad. Sci., 1982, 53-59 [in Russian].

[8] Lanford O.E., A computer assisted proof of the Feigenbaum conjecture, Bull. A.M.S., New Series 6, 1982, V.127, 427-434.

[9] Romanenko E.Yu. and Sharkovsky A.N., Functional-differential equations, which are close to functional ones, in: Asymptotic Methods in the Theory of Nonlinear Oscillations, Kiev, Naukova dumka, 1979, 178-209 [in Russian].

[10] Sharkovsky A.N., Coexistence of cycles of continuous transformation of the straight line into itself, Ukrain. Math. J., 1964, V.16, N 1, 61-70 [in Russian].

[11] Sharkovsky A.N., On asymptotic behavior of solutions of difference-differential equations, in: Differential Equations and Applications: Proc. of the Second Conference, Ruse, Bulgaria, 1982, 835-846 [in Russian].

[12] Sharkovsky A.N., Kolyada S.F., Sivak A.G. and Fedorenko V.V., Dynamics of One-Dimensional Maps, Kiev, Naukova dumka, 1989 [in Russian].

[13] Sharkovsky A.N., Maistrenko Yu.L. and Romanenko E.Yu., Difference Equations and Their Applications, Kluwer Academic Publisher, 1993.

[14] Sharkovsky A.N. and Romanenko E.Yu., Problems of turbulence theory and iteration theory, in: Proc. of the European Conference on Iteration Theory (ECIT-91), World Scientific Publisher, Singapore, 1992, 242-252.

[15] Sharkovsky A.N. and Sivak A.G., Universal order and universal rate of solution bifurcations of difference-differential equations, in: Approximate and Qualitative Methods of the Theory of Differential and Functional-Differential Equations, Kiev, Inst Math. Ukrain. Acad. Sci., 1983, 98-106 [in Russian].

[16] Świątek G., Hyperbolicity is dense in the real quadratic family, SUNY Stony Brook preprint, 1992, $\mathrm{N} 10$. 